\title{
Fourier transform differences and averaged similarities in diatoms
}

\author{
Silverio P. Almeida and Hitoshi Fujii
}

\begin{abstract}
Phase contrast photographs of diatoms are characterized from their Fourier transform taken through an optical diffractometer. The system output is placed on line to a PDP11/40 providing digital subtraction of two output spectral distributions due to different species. Differences obtained in this manner are used for characterizing various species. An average Fourier transform taken through coherent additions is also analyzed to find common features in a given set of diatoms.
\end{abstract}

\section{Introduction}

Optical transforms are well known for providing important information concerning the structure of small objects. ${ }^{1-4}$ The morphology of an object gives rise to a Fraunhofer diffraction pattern whose image contains spatial frequencies characteristic of the components which make up that object. The intensity of light diffracted into the various spatial frequencies is an indication of the extent with which that feature exists in the object. Information on an object's size and shape can be determined from analysis of its diffraction pattern, which, in turn, can be used to find the difference and similarity of the object with respect to some other one.

Diatoms, i.e., algae, have been chosen here for the analysis. The size of diatoms used for this study is about $10 \mu \mathrm{m}$. They are phase structures and have been photographed through a phase contrast microscope to provide $35-\mathrm{mm}$ transparencies used as input to the optical diffractometer. Diffraction patterns for different diatoms are photographed, digitized, and compared with each other. Various combinations of the digitized patterns are then subtracted and their difference plotted. Analysis of these differences provides information characteristic of given diatom species or genus. In addition, optical transforms for different sized diatoms belonging to a certain species are superimposed coherently and analyzed to find a common shape characterizing that species.

The authors are with Virginia Polytechnic Institute and State University, Physics Department, Blacksburg, Virginia 24061.

Received 9 January 1979.

0003-6935/79/101663-05\$00.50/0.

(C) 1979 Optical Society of America.

\section{Optical Fourier Transforms}

\section{A. Optical Diffractometer}

The optical transforms were obtained with the diffractometer shown in Fig. 1. The system shown uses an argon laser to provide uniform coherent illumination for the input transparency, which is located in the front focal plane $P_{1}$ of lens $L_{1}$. The recorded phase contrast image is placed in a liquid cell to remove phase variations in the transparency. With these conditions the Fourier transform of the input function $f(x, y)$ is obtained in the back focal plane $P_{2}$ of $L_{1}$. The field amplitude distribution is given ${ }^{5}$

$$
\Psi(\zeta, \eta)=C \iint_{-\infty}^{\infty} f(x, y) \exp \left[-i \frac{k}{f}(\zeta x+\eta y)\right] d x d y,
$$

where $C=$ constant,

$$
\begin{aligned}
k & =2 \pi / \lambda, \text { and } \\
f & =\text { focal length of lens } L_{1} .
\end{aligned}
$$

The intensity distribution $|\Psi(\zeta, \eta)|^{2}$ or power spectrum of the diatom is photographed and digitized for comparison.

\section{B. Averaged Fourier Transforms of Similar Features}

Normally biological input patterns are never entirely the same. For example, diatoms belonging to the same species are quite similar in shape, but can be different in size and their stria pattern [see Fig. 2(a)]. Therefore, it is sometimes more important to find similarities rather than differences for the pattern recognition or classification of such biological specimens.

Techniques of coherent averaging have been studied and used for particle-size estimation. 6,7 The power spectrums of $N$-randomly distributed disks with various sizes were analyzed to determine their average size. In this section, however, we introduce a slightly different formula for the coherent averaging, since the objective 


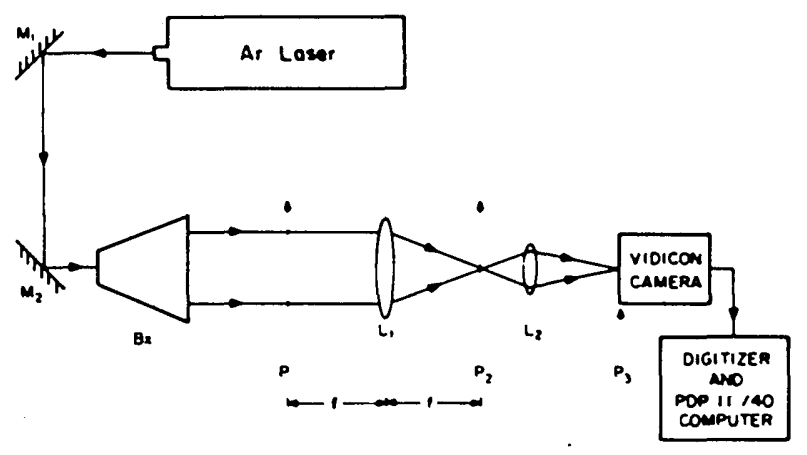

Fig. 1. Optical diffractometer on line to a vidicon and PDP11-40 computer.

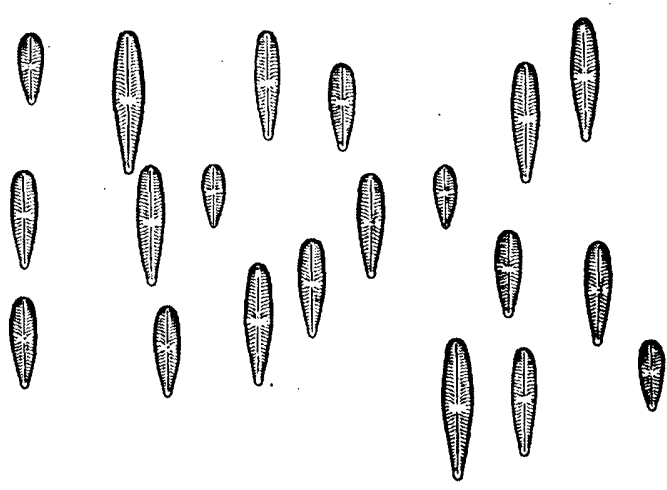

(a)

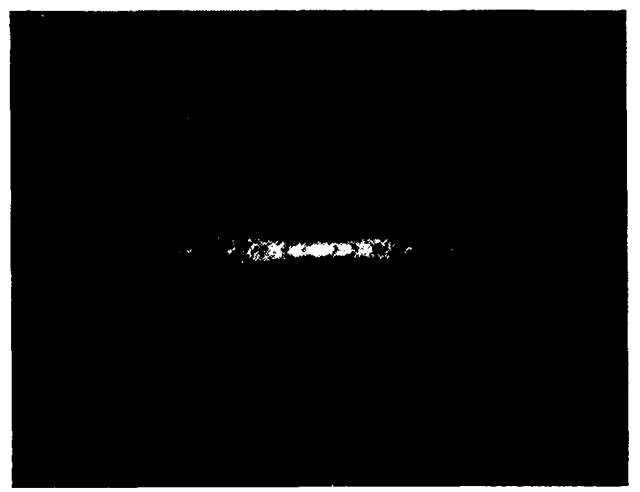

(b)

Fig. 2. (a) Random size distribution for 20 diatoms belonging to the same species and having the same orientation; (b) the averaged power spectrum of (a).

here is to find similarities in a given set of patterns ${ }^{8}$ rather than determine some average dimensions.

Consider an input made up of $N$-randomly distributed functions,

$$
f(x, y)=\sum_{n=1}^{N} f_{n}\left(x-x_{n}, y-y_{n}\right)
$$

We assume that each function has a certain shape in common and is in the same orientation, i.e.,

$$
f_{n}\left(x-x_{n}, y-y_{n}\right)=u\left(x-x_{n}, y-y_{n}\right)+v_{n}\left(x-x_{n}, y-y_{n}\right),
$$

where $u(.$.$) and v_{n}(\ldots)$ stand for the common shape and remaining differences. The amplitude distribution $\Psi(\zeta, \eta)$ in plane $P_{2}$ that is the Fourier transform of the composite input is then given by substituting Eqs. (2) and (3) into Eq. (1):

$$
\begin{aligned}
\Psi(\zeta, \eta)= & C \sum_{n=1}^{N} \exp \left[-i \frac{k}{f}\left(x_{n} \zeta+y_{n} \eta\right)\right] \\
& \times\left\{\iint_{-\infty}^{\infty} u\left(x^{\prime}, y^{\prime}\right) \exp \left[-i \frac{k}{f}\left(\zeta x^{\prime}+\eta y^{\prime}\right)\right] d x^{\prime} d y^{\prime}\right. \\
& \left.+\iint_{-\infty}^{\infty} v_{n}\left(x^{\prime}, y^{\prime}\right) \exp \left[-i \frac{k}{f}\left(\zeta x^{\prime}+\eta y^{\prime}\right)\right] d x^{\prime} d y^{\prime}\right\} \\
= & C \sum_{n=1}^{N} \exp \left[-i \frac{k}{f}\left(x_{n} \zeta+y_{n} \eta\right)\right]\left[U(\zeta, \eta)+V_{n}(\zeta, \eta)\right],
\end{aligned}
$$

where $U(\zeta, \eta)$ and $V_{n}(\zeta, \eta)$ are Fourier transforms of $u\left(x^{\prime}, y^{\prime}\right)$ and $v_{n}\left(x^{\prime}, y^{\prime}\right), x^{\prime}=x-x_{n}$ and $y^{\prime}=y-y_{n}$. Then the power spectrum of the input is expressed by

$$
\begin{aligned}
|\Psi(\zeta, \eta)|^{2}= & C^{2} \sum_{n=1}^{N} \sum_{m=1}^{N} \exp \left\{-i \frac{k}{f}\left[\left(x_{n}-x_{m}\right) \zeta+\left(y_{n}-y_{m}\right) \eta\right]\right\} \\
& \cdot\left[|U(\zeta, \eta)|^{2}+U(\zeta, \eta) V_{m}^{*}(\zeta, \eta)+U^{*}(\zeta, \eta) V_{n}(\zeta, \eta)\right. \\
& \left.+V_{n}(\zeta, \eta) V_{m}^{*}(\zeta, \eta)\right] .
\end{aligned}
$$

Then

$$
\begin{aligned}
|\Psi(\zeta, \eta)|^{2}= & C^{2}\left\{N|U(\zeta, \eta)|^{2}+\sum_{n \neq m}^{N} \sum^{N}|U(\zeta, \eta)|^{2}\right. \\
& \times \exp \left\{-i \frac{k}{f}\left[\left(x_{n}-x_{m}\right) \zeta+\left(y_{n}-y_{m}\right) \eta\right]\right\} \\
& +\sum_{n=1}^{N} \sum_{m=1}^{N}\left[u(\zeta, \eta) V_{m}^{*}(\zeta, \eta)+U^{*}(\zeta, \eta) V_{n}(\zeta, \eta)\right. \\
& \left.+V_{n}(\zeta, \eta) V_{m}^{*}(\zeta, \eta)\right] \\
& \left.\times \exp \left\{-i \frac{k}{f}\left[\left(x_{n}-x_{m}\right) \zeta+\left(y_{n}-y_{m}\right) \eta\right]\right\}\right] .
\end{aligned}
$$

The first term $(m=n)$ is a superposition of the power spectrum of the common shape in a given set of diatoms. In the second summation $(m \neq n)$ all terms vary rapidly if each input pattern is separated far enough from neighboring ones. This corresponds to a modulation of the common spectrum by higher spatial frequency fringes with random orientations. These terms appear as a speckle noise except at the center of the spectrum $(\zeta, \eta)=(0,0)$ where a strong peak is produced by an in phase superposition of all the fringes. In the third summation most of the terms may be negligible since neither the $U(\zeta, \eta), V_{n, m}(\zeta, \eta)$ nor the $V_{n}(\zeta, \eta), V_{m}(\zeta, \eta)$ spatial frequencies fully overlap. What remains is

$$
\sum_{n=1}^{N}\left|V_{n}(\zeta, \eta)\right|^{2}
$$

which is the sum of the dissimilar shape Fourier components. The resulting power spectrum in plane $P_{2}$ is, therefore, composed of contributions from the common shape in the objects together with a broadband spectral distribution due to the remaining parts of the objects plus some speckle noise and a strong peak in the center. 

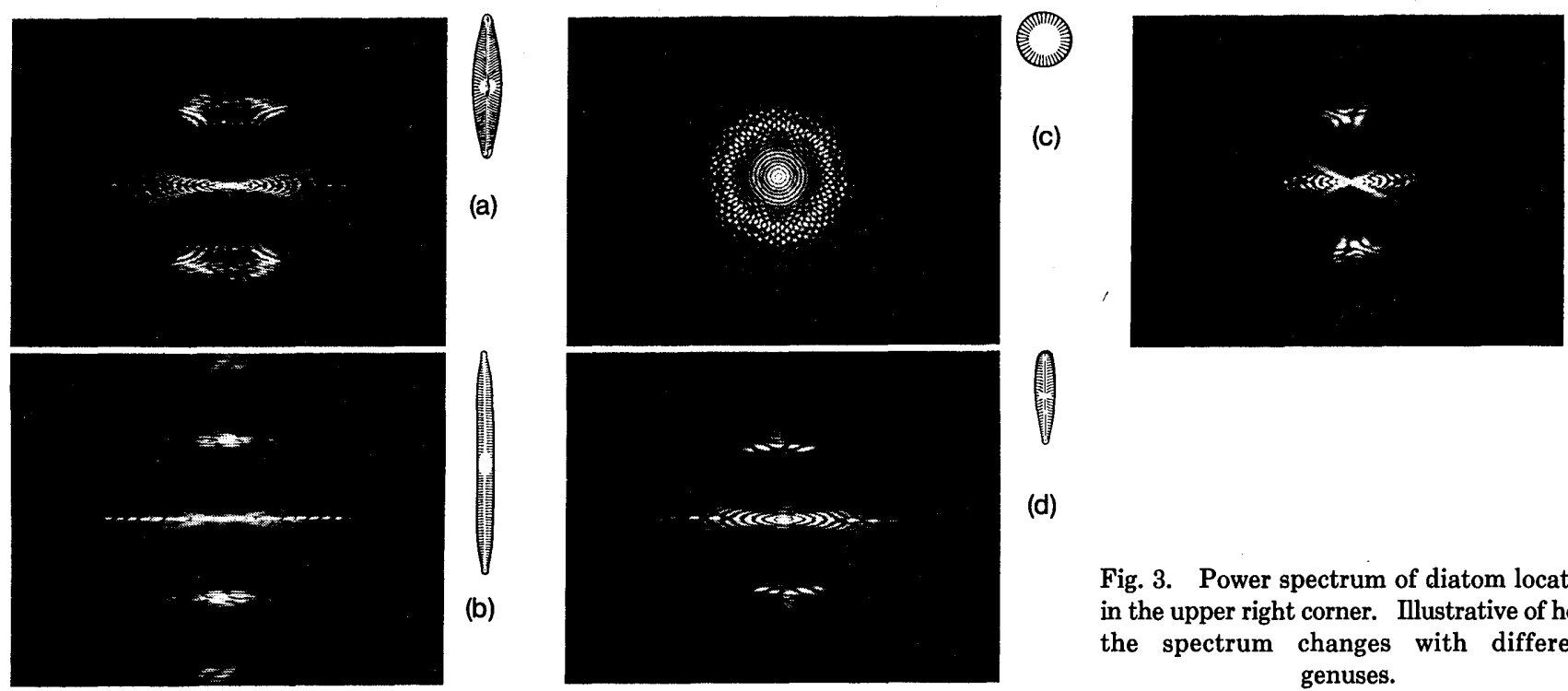

Accordingly, if we could find a definite pattern in the power spectrum of a given set of diatoms in a species, we would like to use it to characterize that species.

\section{Results and Discussion}

Figures 3(a)-(e) are examples of the photographs of power spectrums taken in the plane $P_{2}$ for various input diatoms, which are shown at the top right corner of each print. They belong to different genuses having various morphological features.

The diatom in Fig. 3(a) belongs to genus Navicula whose shape is usually rhombic with two axes of symmetry. The power spectrum thus has two cross lines having periodic structure which is caused by the interference between the pair of straight lines (see the edge of the diatom). The gratinglike stria pattern produces higher frequencies distributed along the vertical axis of the diffraction figure. Since this species Navicula virdula has a radial stria at the middle, those higher frequencies spread also horizontally (see the first and second diffraction orders).

Figure 3(b) shows the case of Synedra. This genus has usually a slender shape with two axes of symmetry. The diffraction pattern is similar to that of a double slit. Since this species Synedra ulna has a parallel stria pattern, the higher diffraction orders distribute close to the vertical axis. (c) is for Cyclotella Meneghiniana, which belongs to the genus Cyclotella. The diffraction pattern shows the concentric circular structure easily recognizable among other genuses. (d) is the genus Gomphonema having narrow egg shape with a major axis of symmetry. The round top and wedged bottom half form a mixture of concentric ellipses and two cross lines. The final example (e) Cymbella has a semicircular shape with a minor axis of symmetry. The diffraction pattern shows both concentric ellipses and two strong cross lines which come up from straight parts of the arc (see the top and bottom ends of the arc). However, it is noticed that the angle of lines is much larger than that in Figs. 3(a) and 3(d). Also the higher

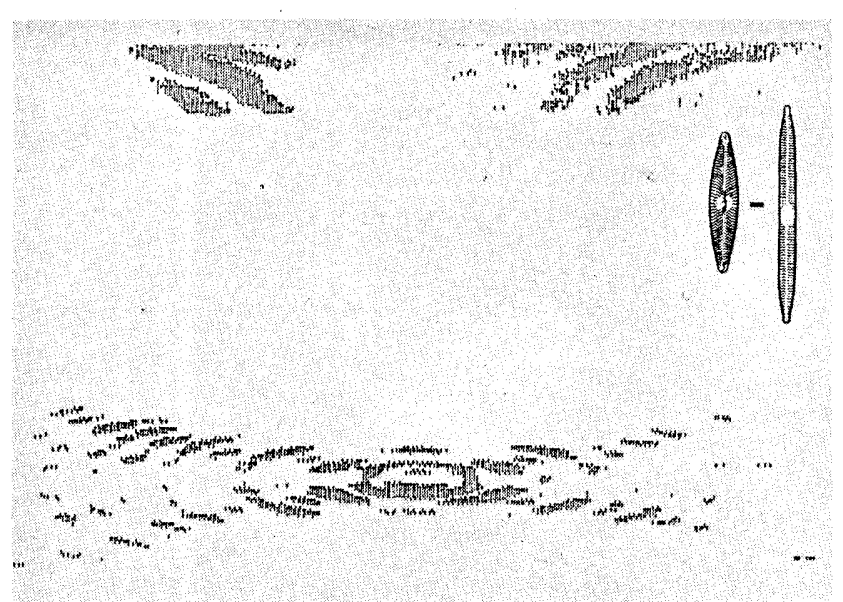

Fig. 3. Power spectrum of diatom located in the upper right corner. Illustrative of how the spectrum changes with different genuses. (a)

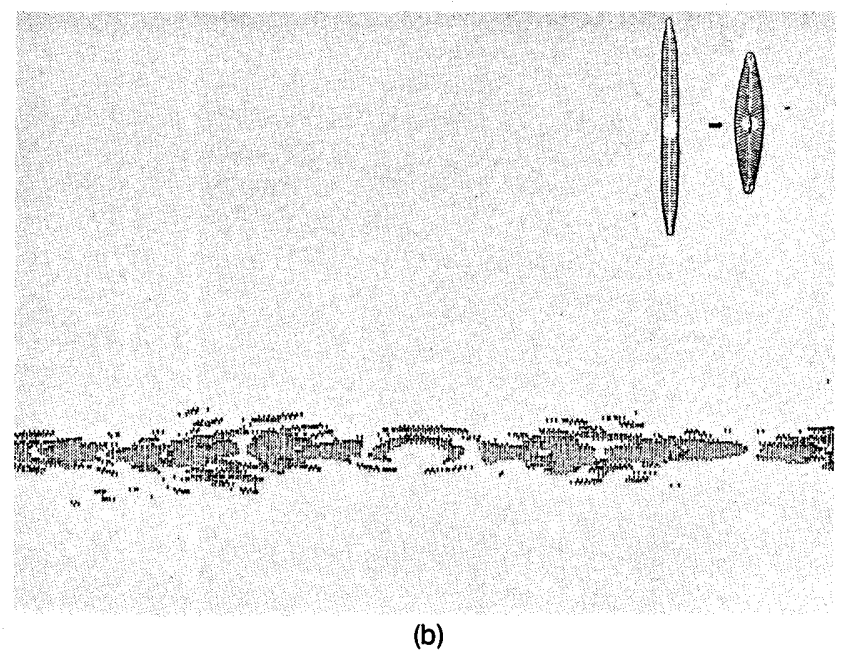

Fig. 4. (a) Plot of the digital subtraction of the two diatoms shown. The result reveals the positive contribution of the diatom located on the left. (b) Plot of the digital subtraction of the two diatoms shown. Reversed order from (a). 
diffraction orders are spaced differently due to the coarse structure of the stria.

These features of diffraction patterns are more clearly seen from their digital subtraction. The image intensity distribution in the plane $P_{3}$ is digitized with a point resolution of $256 \times 384$ pixels/frame into 64 gray levels and stored in a PDP11/40 computer memory. By subtracting one image from the other and plotting the positive part, we obtain the remaining spectral distribution existing only in the original image. Figures 4 and 5 show some of the results for different pairs where only the upper half of the image is magnified and plot-

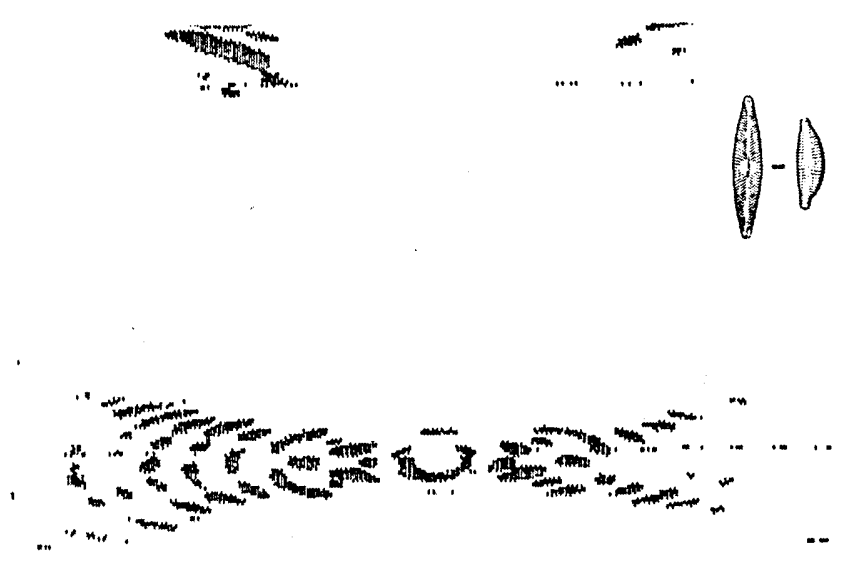

(a)
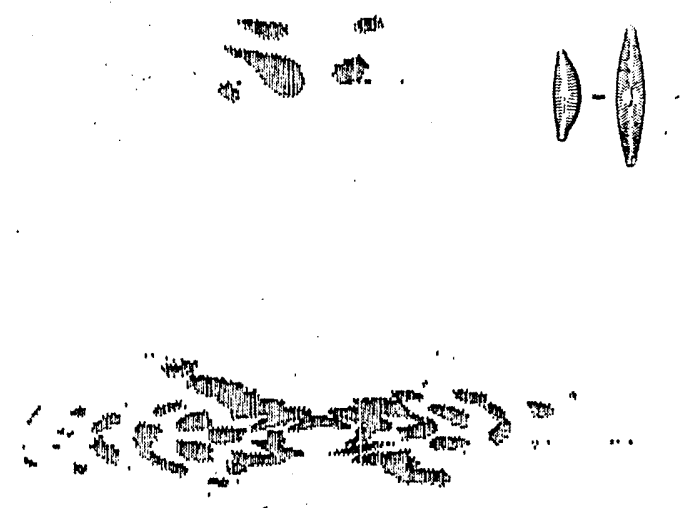

(b)

Fig. 5. Similar plots as shown in Figs. 4(a) and 4(b) only using a different pair of diatoms. ted with vertical line scan. Figure 4(a) is the subtraction Navicula-Synedra which characterizes the shape of Navicula. Figure 4(b) is for the opposite calculation showing the characteristics of Synedra with strong intensity distribution along the horizontal axis. Figure 5 is the similar case for (a) Navicula and (b) Cymbella. It is again seen that Navicula produces two series of bright spots crossing with a narrower angle than that by Cymbella.

As we have shown so far, the power spectra of those diatoms contain valuable information on their morphological features. Such information, in turn, allows us to recognize and classify the diatoms or to find a variety of them. Considering an actual situation of such biological pattern recognition, we did some preliminary experiments on the average power spectrum. Based on the theoretical results in the previous section, we prepared an input of $N$-randomly distributed diatoms belonging to the same species but of different individuals all oriented in the same direction [see Fig. 2(a)]. The purpose of this study is to obtain averaged morphological features of each species and generalize the previous analysis of a single specimen.

Figure 2(a) is the input for the species Gomphonema olvaceum composed of two sets of ten different sized diatoms. It is seen that the actual specimens have considerable variety in shape, size, and stria. Nevertheless, their basic structure, horseshoelike top and wedged bottom, is common in all individuals. The spectrum of these similar features appears clearly in Fig. 2(b) with a noisy background.

Figures 6 and 7 are the results of similar experiments for other species used in Figs. 3(a) and 3(e). Comparing these figures with Figs. 3(a) and 3(e), the averaged power spectrum contains more common morphological features, which are useful to characterize the actual biological specimens.

\section{Conclusion}

The study of diffraction patterns has provided useful information for morphology studies. Since diatoms have relatively uniform, simple, and symmetric structures, beautiful diffraction patterns were obtained which allow us to characterize their morphological features. Digital subtractions and a coherent averaging technique have shown some quantitative results of the spectral distribution for the more general case. Differences obtained in this manner are useful either for direct power spectrum analysis with wedge-ring detectors ${ }^{4}$ or in masking matched spatial filters. ${ }^{9,10}$

The authors thank Guy Indebetouw for helpful discussions and Jean-Marc Fournier and Peter Pryfogle for help in the preparation of the biological input. This research was supported under the National Science Foundation ASRA Division under grant ENV7203581-A05. 


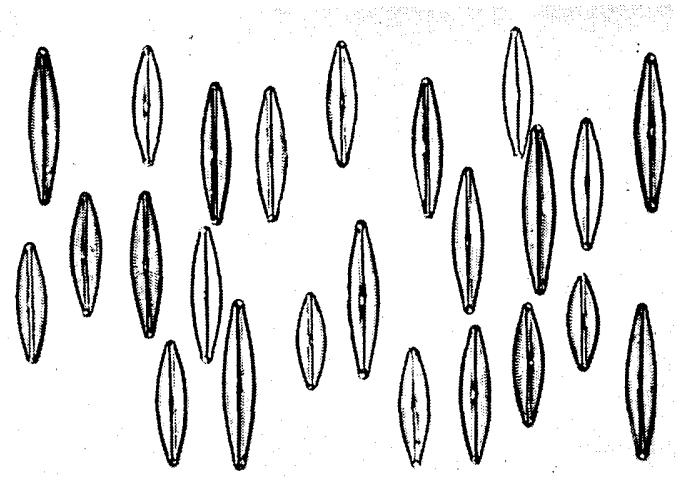

(a)

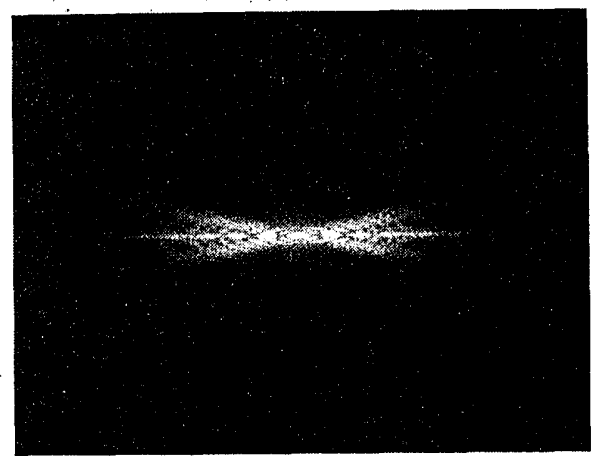

(b)

Fig. 6. (a) Random size distribution for 24 diatoms belonging to the same species and having the same orientation; (b) the averaged power spectrum of (a). These species are different from those shown in Fig. 2(a).

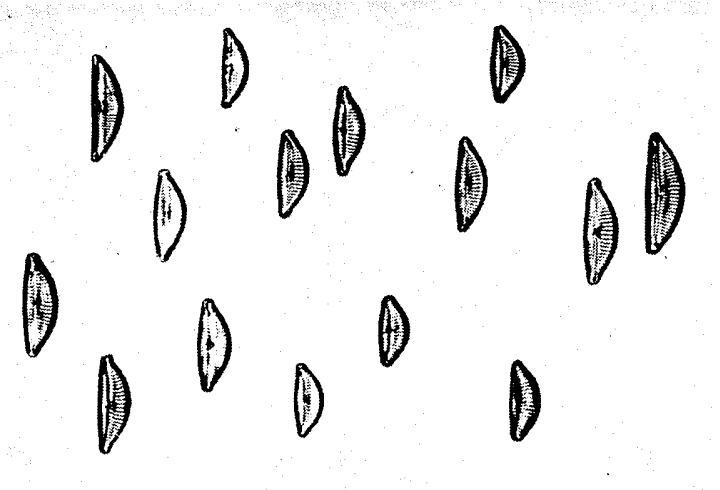

(a)

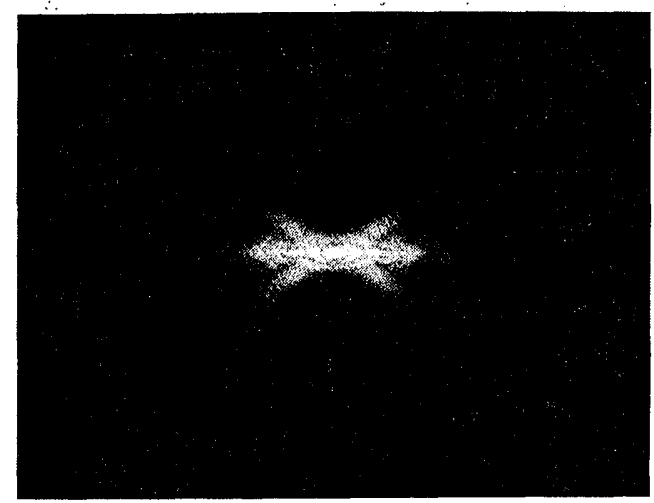

(b)

Fig. 7. (a) Random size distribution for 15 diatoms belonging to a third species, different from those of Figs. 2(a) and 6(a). These are again oriented in the same direction. They possess a symmetry about one axis as opposed to the two-axis symmetry found in diatoms of Fig. 6(a). (b) The averaged power spectrum of the diatoms shown in (a).

\section{References}

1. H. Lipson, Ed., Optical Transforms (Academic, London, 1972).

2. G. Harburn, C. A. Taylor, and T. R. Welberry, Atlas of Optical Transforms (Cornell U. P., Ithaca, 1975).

3. B. J. Thompson, "Optical Transforms and Coherent Processing Systems," in Optical Data Processing, D. Casasent, Ed. (Springer, New York, 1978), Vol. 23, p. 17.

4. B. Pernick, R. E. Kopp, J. Lisa, J. Mendelsohn, H. Stone, and R. Wohlers, Appl. Opt. 17, 21 (1978).

5. J. W. Goodman, Introduction to Fourier Optics (McGraw-Hill, New York, 1968).

6. H. Stark, D. Lee, and B. Dimitriadis, J. Opt. Soc. Am. 65, 1436 (1975).

7. J. Fleuret, H. Maitre, and J. F. Thery, "Analysis of the Diffraction Spectrum of a Population of Particles," in 1st European Congress on Optics Applied to Metrology, M. Grosmann and P. Meyrueis, Eds. (SPIE, New York, 1977), Vol. 136.

8. J. C. Vienot, J. Duvernoy, G. Tribillon, and J. L. Tribillon, Appl. Opt. 12, 950 (1973).

9. S. P. Almeida and J. K. T. Eu, Appl. Opt. 15, 510 (1976).

10. H. Fujii and S. P. Almeida, Appl. Opt. 18, 1659 (1979). 